\title{
Travelling wave solutions of the generalized Benjamin-Bona-Mahony equation
}

\author{
P. G. Estévez \\ Departamento de Física Fundamental, Área de Física Teórica, Universidad de \\ Salamanca, 37008 Salamanca, Spain \\ Ş. Kuru*, J. Negro and L. M. Nieto \\ Departamento de Física Teórica, Atómica y Óptica, Universidad de Valladolid, \\ 47071 Valladolid, Spain
}

\begin{abstract}
A class of particular travelling wave solutions of the generalized Benjamin-BonaMahony equation is studied systematically using the factorization technique. Then, the general travelling wave solutions of Benjamin-Bona-Mahony equation, and of its modified version, are also recovered.

Pacs:05.45.Yv, 52.35.Mw, 52.35.Sb, 02.30.Jr
\end{abstract}

\section{Introduction}

The generalized BBM (Benjamin-Bona-Mahony) equation has a higher order nonlinearity of the form

$$
u_{t}+u_{x}+a u^{n} u_{x}+u_{x x t}=0, \quad n \geq 1,
$$

where $a$ is constant. The case $n=1$ corresponds to the BBM equation

$$
u_{t}+u_{x}+a u u_{x}+u_{x x t}=0
$$

which was first proposed in 1972 by Benjamin et al [1]. This equation is an alternative to the Korteweg-de Vries (KdV) equation, and describes the unidirectional propagation of small-amplitude long waves on the surface of water in

* On leave of absence from: Department of Physics, Faculty of Science, Ankara University 06100 Ankara, Turkey.

Email address: jnegro@fta.uva.es (J. Negro). 
a channel. The BBM equation is not only convenient for shallow water waves but also for hydromagnetic waves, acoustic waves, and therefore it has more advantages compared with the $\mathrm{KdV}$ equation. When $n=2$, Eq.(1) is called the modified BBM equation

$$
u_{t}+u_{x}+a u^{2} u_{x}+u_{x x t}=0
$$

When looking for travelling wave solutions, the BBM and modified BBM equations can be reduced to ordinary differential equations that possess the Painlevé property and which are integrable in terms of elliptic functions [2,3]. The generalized BBM equation is also integrable in terms of elliptic functions, provided that some restrictions on the parameters are imposed. Recently many methods have been presented to obtain the travelling wave solutions of the generalized BBM equation: the tanh-sech and the sine-cosine methods [4,5], an approach based on balancing principle to obtain some explicit solutions in terms of elliptic function [6], and an extended algebraic method with symbolic computation [7].

Our aim is to investigate systematically the travelling wave solutions of these equations, applying the factorization technique [8,9]. Thus, we will get all the previously known solutions and some new ones, supplying a new general approach. Assuming that the generalized BBM equation has an exact solution in the form of a travelling wave, then it will reduce to a third order ordinary differential equation (ODE). This equation can be integrated trivially to a second order ODE, which can be factorized in two ways: the first one by means of differential operators and the second one by using a first integral (that can also be factorized in terms of first integrals). These factorizations give rise to the same first order ODE that provides the travelling wave solutions of the nonlinear equation. This first order ODE for $n=1$ and $n=2$ is integrable, but for other values of $n$, we can also find some particular solutions by imposing some restrictions on the parameters.

The paper is organized as follows. In section 2, we introduce the factorization technique for nonlinear equations, and we show how to apply it to find the travelling wave solutions of the generalized BBM equation. In section 3, we consider some special cases to get particular solutions of generalized BBM. In section 4, we obtain the solutions of the BBM and modified BBM. Finally, section 5 ends the paper with some conclusions. In the Appendix, we give also some useful information about the elliptic functions that are used in the previous sections. 


\section{Travelling waves of the generalized Benjamin-Bona-Mahony equa- tion}

\subsection{Travelling wave solutions}

Let us assume that Eq. (11) has an exact solution in the form of a travelling wave

$$
u(x, t)=\phi(\xi), \quad \xi=h x-\omega t,
$$

where $h$ and $\omega$ are real constants. If we substitute (4) in Eq. (1), we get

$$
-h^{2} \omega \phi_{\xi \xi \xi}+(h-\omega) \phi_{\xi}+h a \phi^{n} \phi_{\xi}=0
$$

After integrating with respect to $\xi$, we have

$$
\phi_{\xi \xi}-\frac{h-\omega}{h^{2} \omega} \phi-\frac{a}{(n+1) h \omega} \phi^{n+1}=-R,
$$

where $R$ is an integration constant. Let us introduce the following linear transformation of the dependent and independent variables

$$
\xi=h \theta, \quad \phi(\xi)=\left(\frac{c(n+1)}{a}\right)^{1 / n} W(\theta)
$$

where $\theta=x-c t$ and $c=\omega / h$. In this way (6) becomes the nonlinear second order ODE

$$
\frac{d^{2} W}{d \theta^{2}}-W^{n+1}-k W=D
$$

where the new constants are

$$
k=\frac{1-c}{c}, \quad D=-R h^{2}\left(\frac{a}{c(n+1)}\right)^{1 / n} .
$$

Therefore, if we are interested in finding the travelling wave solutions of (11), we have to solve the ODE (18).

\subsection{Factorization of some special type of nonlinear second order ODE}

In this section we will introduce a factorization technique applied to nonlinear second order ODE of the special form

$$
\frac{d^{2} W}{d \theta^{2}}-\beta \frac{d W}{d \theta}+F(W)=0
$$


where $F(W)$ is an arbitrary function of $W$ and $\beta$ is constant. This equation can be factorized as

$$
\left[\frac{d}{d \theta}-f_{2}(W, \theta)\right]\left[\frac{d}{d \theta}-f_{1}(W, \theta)\right] W(\theta)=0
$$

being $f_{1}$ and $f_{2}$ two unknown functions that may depend explicitly on $W$ and $\theta$. In order to find $f_{1}$ and $f_{2}$, we expand (11)

$$
\frac{d^{2} W}{d \theta^{2}}-\left(f_{1}+f_{2}+\frac{\partial f_{1}}{\partial W} W\right) \frac{d W}{d \theta}+f_{1} f_{2} W-W \frac{\partial f_{1}}{\partial \theta}=0
$$

and then comparing with (10), we obtain the following consistency conditions

$$
\begin{aligned}
& f_{1} f_{2}=\frac{F}{W}+\frac{\partial f_{1}}{\partial \theta}, \\
& f_{2}+\frac{\partial\left(W f_{1}\right)}{\partial W}=\beta .
\end{aligned}
$$

If we find a solution for this factorization problem, it will allow us to write a compatible first order ODE

$$
\left[\frac{d}{d \theta}-f_{1}(W, \theta)\right] W(\theta)=0
$$

that provides a (particular) solution [8,9] to the nonlinear ODE (10). In the applications of this paper $f_{1}$ and $f_{2}$ will depend only on $W$.

\subsection{Factorization of the generalized BBM equation}

When we apply the factorization technique described above to Eq. (8), then we have $\beta=0, F(W)=-W^{n+1}-k W-D$, and the consistency conditions given by (13) and (14) take the form

$$
\begin{gathered}
f_{1} f_{2}=-W^{n}-k-D W^{-1}, \\
f_{2}=-f_{1}-W \frac{\partial f_{1}}{\partial W} .
\end{gathered}
$$

Substituting (17) in (16) we get

$$
W f_{1} \frac{\partial f_{1}}{\partial W}+f_{1}^{2}-W^{n}-k-D W^{-1}=0 .
$$


The solutions of this equation are

$$
f_{1}= \pm \sqrt{\frac{2 W^{n}}{n+2}+k+\frac{2 D}{W}+\frac{C}{W^{2}}}
$$

where $C$ is an integration constant. By replacing $f_{1}$ given by (19) in Eq. (15), we have the first order ODE

$$
\frac{d W}{d \theta} \mp \sqrt{\frac{2 W^{n+2}}{n+2}+k W^{2}+2 D W+C}=0 .
$$

As we have already mentioned, when we solve this equation, we get also a particular solution of (8) .

In the special case of the generalized BBM equation, another way to get this result is as follows: if Eq. (8) is multiplied by $2 W^{\prime}$ and integrated, we arrive to

$$
\begin{aligned}
& {\left[\frac{d W}{d \theta}-\sqrt{\frac{2 W^{n+2}}{n+2}+k W^{2}+2 D W+C_{0}}\right]} \\
& \times\left[\frac{d W}{d \theta}+\sqrt{\frac{2 W^{n+2}}{n+2}+k W^{2}+2 D W+C_{0}}\right]=0
\end{aligned}
$$

which is equivalent to (20). Remark that the last equation is a product of functions, while the factorization (11) is a product of operators.

These factorizations are valid for any value of the parameters $(k, n)$ and the integration constants $(C, D)$. However, the integrability of this equation is obtained, in general, only if some constraints are imposed. As a result, it can be said that the factorization does not imply the integrability of a nonlinear equation, but can produce some solutions, under certain parameter restrictions (to be determined later, when solving Eq. (200)).

If we want to guarantee the integrability of (20), the powers of $W$ have to be integer numbers between 0 and 4 , and therefore $n \in\{-1,0,1,2\}[3]$. [Nevertheless, recall that an initial assumption on the generalized BBM equation (1) is $n \geq 1$.] On the other side, equation (8) has the Painlevé property only if the same condition on $n$ is satisfied [3]. In these cases Eq. (8) is integrable in terms of elliptic functions.

In order to find other values of $n$ for which it is possible to find particular solutions, let us make in Eq. (20) the transformation $W=\varphi^{p}, p \neq 0,1$, getting

$$
\left(\frac{d \varphi}{d \theta}\right)^{2}=\frac{2}{(n+2) p^{2}} \varphi^{2+n p}+\frac{k}{p^{2}} \varphi^{2}+\frac{2 D}{p^{2}} \varphi^{2-p}+\frac{C}{p^{2}} \varphi^{2-2 p} .
$$


This equation is of the same type as the initial one (20), hence the same integrability conditions are valid. In this way we have the following additional cases, that will require to impose some conditions on the parameters (with $n \geq 1)$ :

(1) If $C=D=0$, then

$$
p \in\left\{-\frac{2}{n},-\frac{1}{n}, \frac{1}{n}, \frac{2}{n}\right\} .
$$

In the next section it will be proved that $p=1 / n$ and $p=2 / n$ give the same solution $u(x, t)$ of the BBM equation (11), and therefore we consider only the case $p=1 / n$. Then, Eq. (21) takes the form

$$
\left(\frac{d \varphi}{d \theta}\right)^{2}=\frac{2 n^{2}}{(n+2)} \varphi^{3}+k n^{2} \varphi^{2} .
$$

The same discussion applies for the values $p=-1 / n, p=-2 / n$, and choosing $p=-1 / n$, Eq. (21) becomes

$$
\left(\frac{d \varphi}{d \theta}\right)^{2}=\frac{2 n^{2}}{(n+2)} \varphi+k n^{2} \varphi^{2} .
$$

(2) If $C \neq 0$ and $D=0$, we have $n=4$ and two possibilities: either $p=1 / 2$ with

$$
\left(\frac{d \varphi}{d \theta}\right)^{2}=\frac{4}{3} \varphi^{4}+4 k \varphi^{2}+4 C \varphi,
$$

or $p=-1 / 2$ with

$$
\left(\frac{d \varphi}{d \theta}\right)^{2}=\frac{4}{3}+4 k \varphi^{2}+4 C \varphi^{3} .
$$

(3) If $C=0$ and $D \neq 0$, no new solutions appear.

The solutions of equations (22)-(25) can be expressed in terms of elliptic functions. Then, using (44), (77), (9), and recalling $W(\theta)=\varphi^{p}(\theta)$, the particular solution of the generalized BBM equation (1) reads

$$
u(x, t)=\left(\frac{c(n+1)}{a}\right)^{1 / n} \varphi^{p}(x-c t) .
$$

The details are given in the next section.

\section{Solutions of the generalized Benjamin-Bona-Mahony equation}

In this section, using the basic results on elliptic functions shown in the Appendix, we will analyze the solutions of the four equations (22)-(25) obtained 
before, which produce particular solutions of the BBM equation.

\subsection{Case $C=D=0, p=1 / n$}

In this case the third order polynomial of Eq. (22)

$$
f(\varphi)=\frac{2 n^{2}}{(n+2)} \varphi^{3}+k n^{2} \varphi^{2}
$$

has two roots: $\varphi_{0}=0$ (with multiplicity two) and $\varphi_{0}=-k(n+2) / 2$. When we substitute the derivatives of $f(\varphi)$ in terms of $\varphi_{0}$ in Eq. (98), we get the following solution for Eq. (22)

$$
\varphi=\frac{12 \varphi_{0} \wp\left(\theta ; g_{2}, g_{3}\right)+\frac{12 n^{2}}{n+2} \varphi_{0}^{2}+5 k n^{2} \varphi_{0}}{12 \wp\left(\theta ; g_{2}, g_{3}\right)-\frac{6 n^{2}}{n+2} \varphi_{0}-k n^{2}}
$$

where the invariants are

$$
g_{2}=\frac{k^{2} n^{4}}{12}, \quad g_{3}=-\frac{k^{3} n^{6}}{216} .
$$

The root $\varphi_{0}=0$ gives the trivial solution, $\varphi=0$, and the nonzero solution of Eq. (22) can be easily found replacing $\varphi_{0}=-k(n+2) / 2$ in (28):

$$
\varphi=\frac{k(n+2)}{4}\left(\frac{k n^{2}-12 \wp\left(\theta ; g_{2}, g_{3}\right)}{k n^{2}+6 \wp\left(\theta ; g_{2}, g_{3}\right)}\right)
$$

or using relation (99) in (30)

$$
\varphi=\frac{2(n+2)}{n^{2}} \wp\left(\theta+\omega ; g_{2}, g_{3}\right)-\frac{k(n+2)}{6}
$$

being $\omega$ the half-period. For the values $g_{2}$ and $g_{3}$ of (29), the discriminant defined by (101) is equal to zero. Hence, we have both solitary wave and periodic solutions. When we use (102) in (30), we have the solitary wave solution

$$
\varphi=-\frac{k(n+2)}{2} \operatorname{sech}^{2}\left[\frac{n}{2} \sqrt{k} \theta\right]
$$

for $0<c<1$. We get the same result by making use of (31) with the halfperiod $\omega^{\prime}=(i \pi / \sqrt{k} n)$. Using half-angle formulas for $\cosh x$, solution (32) can also be written as

$$
\varphi=-k(n+2) \frac{1}{1+\cosh [n \sqrt{k} \theta]} .
$$

When we use (103) in (30), we have the periodic solution

$$
\varphi=\frac{k(n+2)}{2} \sec ^{2}\left[\frac{n}{2} \sqrt{-k} \theta\right]
$$


for $c>1$. The same result can be obtained from (31) with the half-period $\omega=(\pi / \sqrt{-k} n)$. This solution can also be rewritten in the form

$$
\varphi=k(n+2) \frac{1}{1+\cos [n \sqrt{-k} \theta]}
$$

Finally, substituting (32)-(35) in (26) and taking into account (9), we get the following solutions of Eq. (11)

$$
\begin{aligned}
u(x, t) & =\left(\frac{(n+1)(n+2)(c-1) / a}{1+\cosh \left[n \sqrt{\frac{1-c}{c}}(x-c t)\right]}\right)^{1 / n} \\
& =\left(\frac{(n+1)(n+2)(c-1)}{2 a}\right)^{1 / n}\left(\operatorname{sech}^{2}\left[\frac{n}{2} \sqrt{\frac{1-c}{c}}(x-c t)\right]\right)^{1 / n}
\end{aligned}
$$

(solitary waves) for $c<1$, and

$$
\begin{aligned}
u(x, t) & =\left(\frac{(n+1)(n+2)(1-c) / a}{1+\cos \left[n \sqrt{\frac{c-1}{c}}(x-c t)\right]}\right)^{1 / n} \\
& =\left(\frac{(n+1)(n+2)(1-c)}{2 a}\right)^{1 / n}\left(\sec ^{2}\left[\frac{n}{2} \sqrt{\frac{c-1}{c}}(x-c t)\right]\right)^{1 / n}
\end{aligned}
$$

(periodic) for $c>1$.

We will show now that, as it was mentioned before, by choosing in Eq. (21) $p=2 / n$ instead of $p=1 / n$, we will recover the same solutions for (1). In this case, Eq. (21) becomes

$$
\left(\frac{d \varphi}{d \theta}\right)^{2}=\frac{n^{2}}{2(n+2)} \varphi^{4}+\frac{k n^{2}}{4} \varphi^{2}
$$

and the forth order polynomial has three different roots: $\varphi_{0}=0$ (with multiplicity two) and

$$
\varphi_{0}= \pm \sqrt{\frac{-k(n+2)}{2}} .
$$

From (98), the solution of Eq. (38) for each root $\left(\varphi_{0}\right)$ can be found

$$
\varphi=\frac{48 \varphi_{0} \wp\left(\theta ; g_{2}, g_{3}\right)+\frac{12 n^{2}}{n+2} \varphi_{0}^{3}+5 k n^{2} \varphi_{0}}{48 \wp\left(\theta ; g_{2}, g_{3}\right)-k n^{2}-\frac{12 n^{2}}{n+2} \varphi_{0}^{2}}
$$


where the invariants are given by

$$
g_{2}=\frac{k^{2} n^{4}}{192}, \quad g_{3}=-\frac{k^{3} n^{6}}{13824}
$$

When we choose $\varphi_{0}=0$, we have again the trivial solution $\varphi=0$, but if we use the roots given in Eq. (39), we get the solutions of Eq. (38)

$$
\varphi^{ \pm}=\mp \sqrt{\frac{-k(n+2)}{2}} \frac{k n^{2}-48 \wp\left(\theta ; g_{2}, g_{3}\right)}{5 k n^{2}+48 \wp\left(\theta ; g_{2}, g_{3}\right)} \text {. }
$$

For $g_{2}$ and $g_{3}$ given in (41), the discriminant is equal to zero, $\Delta=0$, so the Weierstrass function degenerates into trigonometric and hyperbolic functions. Then, taking into account (102) and (103), it is easy to see that the solutions (42) give rise to the same solitary wave and periodic solutions of Eq. (11) as (36) and (37).

\subsection{Case $C=D=0, p=-1 / n$}

Here we have the second order polynomial

$$
f(\varphi)=\frac{2 n^{2}}{(n+2)} \varphi+k n^{2} \varphi^{2}
$$

with two roots: $\varphi_{0}=0$ and

$$
\varphi_{0}=-\frac{2}{k(n+2)}
$$

The expression for the solutions of (23) in terms of $\varphi_{0}$ is

$$
\varphi=\frac{12 \varphi_{0} \wp\left(\theta ; g_{2}, g_{3}\right)+\frac{6 n^{2}}{n+2}+5 k n^{2} \varphi_{0}}{12 \wp\left(\theta ; g_{2}, g_{3}\right)-k n^{2}}
$$

where the invariants are given by (29). Taking the root $\varphi_{0}=0$ in (45), we get

$$
\varphi=\frac{6 n^{2}}{12(n+2) \wp\left(\theta ; g_{2}, g_{3}\right)-k n^{2}(n+2)} .
$$

Since the discriminant is equal to zero for $g_{2}$ and $g_{3}$ given by (29), we can express the Weierstrass function in terms of trigonometric and hyperbolic functions. Thus, substituting (102) in (46), the solitary wave solution can be written

$$
\varphi=-\frac{2}{k(n+2)} \sinh ^{2}\left[\frac{n}{2} \sqrt{k} \theta\right]
$$


for $c<1$, and using (103) in (46), we have the periodic solution

$$
\varphi=\frac{2}{k(n+2)} \sin ^{2}\left[\frac{n}{2} \sqrt{-k} \theta\right]
$$

for $c>1$.

When we take the second root (44) in (45), we have

$$
\varphi=\frac{4 k n^{2}+24 \wp\left(\theta ; g_{2}, g_{3}\right)}{k(n+2)\left(k n^{2}-12(n+2) \wp\left(\theta ; g_{2}, g_{3}\right)\right)} \text {. }
$$

Having in mind the degenerate cases of the Weierstrass function (shown in the Appendix), this solution can be expressed as

$$
\varphi=-\frac{2}{k(n+2)} \cosh ^{2}\left[\frac{n}{2} \sqrt{k} \theta\right]
$$

for $c<1$ and

$$
\varphi=\frac{2}{k(n+2)} \cos ^{2}\left[\frac{n}{2} \sqrt{-k} \theta\right]
$$

for $c>1$. Substituting (47)-(50) in (26) and taking into account (9), we get the solutions of Eq. (11) which were given by (36) and (37). In addition, it can be also proved that the choice $p=-2 / n$ in Eq. (21) gives exactly the same solutions for Eq. (1).

\subsection{Case $C \neq 0, D=0, n=4, p=1 / 2$}

Now the quartic polynomial

$$
f(\varphi)=\frac{4}{3} \varphi^{4}+4 k \varphi^{2}+4 C \varphi
$$

has four roots. Substituting the first and second derivative of (51) in terms of $\varphi_{0}$ in (98), we have the general expression for the solutions

$$
\varphi=\frac{3 \varphi_{0} \wp\left(\theta ; g_{2}, g_{3}\right)+2 \varphi_{0}^{3}+5 k \varphi_{0}+3 C}{3 \wp\left(\theta ; g_{2}, g_{3}\right)-2 \varphi_{0}^{2}-k}
$$

where the invariants are given by

$$
g_{2}=\frac{4 k^{2}}{3}, \quad g_{3}=-\frac{8 k^{3}}{27}-\frac{4 C^{2}}{3} .
$$

When we take the simplest root of $f(\varphi), \varphi_{0}=0$, the solution of Eq. (24) is

$$
\varphi=\frac{C}{\wp\left(\theta ; g_{2}, g_{3}\right)-k / 3}
$$


Replacing (54) and (91) in (26), we get the particular solution of Eq. (11)

$$
u(x, t)=\left(\sqrt{\frac{5 c}{a}} \frac{3 c C}{3 c \wp(x-c t)+c-1}\right)^{1 / 2} .
$$

Using the other roots of (51) in Eq. (198), we can get the other solutions, that become trigonometric and hyperbolic, like the solutions of the case 3.1 for $C=0$.

\subsection{Case $C \neq 0, D=0, n=4, p=-1 / 2$}

In this case we have the third order polynomial

$$
f(\varphi)=4 C \varphi^{3}+4 k \varphi^{2}+\frac{4}{3}
$$

has three roots. Here we will not give the roots of this polynomial, since they are a bit cumbersome. But, substituting the first and second derivative of (56) in terms of $\varphi_{0}$ in (98), we have the general expression for the solutions

$$
\varphi=\frac{3 \varphi_{0} \wp\left(\theta ; g_{2}, g_{3}\right)+5 k \varphi_{0}+6 C \varphi_{0}^{2}}{3 \wp\left(\theta ; g_{2}, g_{3}\right)+3 C \varphi_{0}-k}
$$

where the invariants given by (53) and the discriminant $\Delta \neq 0$.

We also notice that the choice $n=4, p=-1 / 2$ gives rise to the same particular solution (55) for Eq. (1) and the other solutions can be found by the same procedure as in the above cases.

\section{Solutions of the BBM and modified BBM}

In this section we will consider the solutions obtained for $n=1,2$ when the integration constant $D$ of Eq. (8) is such that $D \neq 0$ (to avoid confusion, in this section we will use the notation $D \equiv D_{n}$, because, as we will immediately see, this constant is chosen to be dependent on $n$ ).

First of all, let us transform Eq. (8) through the change of function $W(\theta)=$ $U(\theta)+\delta$ into 


$$
\begin{aligned}
& \frac{d^{2} U}{d \theta^{2}}-\left(U^{n+1}+\frac{(n+1) !}{n !} U^{n} \delta+\cdots\right. \\
& \left.\quad+\frac{(n+1) !}{2(n-1) !} U^{n-1} \delta^{2}+\ldots+\frac{(n+1) !}{n !} U \delta^{n}\right)=k U
\end{aligned}
$$

where the integration constant $D_{n}$ has been chosen as

$$
D_{n}=-k \delta-\delta^{n+1}
$$

Remark that this change of variable does not give any restriction on the solution of (8).

\subsection{BBM equation $(n=1)$}

We can find the solutions of Eq. (2) when the integration constant is non-zero, $D \neq 0$, in Eq. (8). To do this, first we take $n=1$ and $\delta \neq 0$ in Eq. (58)

$$
\frac{d^{2} U}{d \theta^{2}}-U^{2}+(2 \delta-k) U=0
$$

with

$$
D_{1}=-k \delta-\delta^{2}
$$

For this case, the third order polynomial is

$$
f(U)=\frac{2}{3} U^{3}-(2 \delta-k) U^{2}+C_{1}
$$

with three different roots. When we follow the same procedure as mentioned above, we get the solutions of the second order ODE (60) for all roots

$$
U(\theta)=-\frac{5(k-2 \delta) U_{0}+4 U_{0}^{2}+12 U_{0} \wp\left(\theta ; g_{2}, g_{3}\right)}{(k-2 \delta)+2 U_{0}-12 \wp\left(\theta ; g_{2}, g_{3}\right)}
$$

where

$$
g_{2}=\frac{(k-2 \delta)^{2}}{12}, \quad g_{3}=-\frac{(k-2 \delta)^{3}}{216}-\frac{C_{1}}{36},
$$

and $\Delta \neq 0$. Then the solutions of Eq. (2) can be found from the relation

$$
u(x, t)=\frac{2 c}{a}(U(\theta)+\delta) .
$$

When we choose $C_{1}=0$, the polynomial (62) has two different roots: 0 (with multiplicity two ) and $U_{0}=-3(k-2 \delta) / 2$. For the nonzero root we have the following solution

$$
U(\theta)=6 \wp\left(\theta+\omega ; g_{2}, g_{3}\right)-\frac{(k-2 \delta)}{2}
$$


where

$$
g_{2}=\frac{(k-2 \delta)^{2}}{12}, \quad g_{3}=-\frac{(k-2 \delta)^{3}}{216}
$$

with $\Delta=0$. Therefore, we can express the Weierstrass function as hyperbolic (102) and trigonometric (103) forms. Then, substituting $U(\theta)$ given by (65) in (64), having in mind the simplified form of the Weierstrass function, and choosing $\delta=3 k / 4$ where $k=(1-c) / c, \theta=x-c t$, we have solitary wave (dark soliton) solutions for $c>1$

$$
u(x, t)=\frac{3(c-1)}{2 a} \tanh ^{2}\left[\frac{1}{2} \sqrt{\frac{c-1}{2 c}}(x-c t)\right]
$$

and periodic singular solutions for $c<1$

$$
u(x, t)=\frac{3(1-c)}{2 a} \tan ^{2}\left[\frac{1}{2} \sqrt{\frac{1-c}{2 c}}(x-c t)\right] .
$$

Here we can also consider the special value $\delta=0$. In this case we have

$$
\left(\frac{d U}{d \theta}\right)^{2}=\frac{2}{3} U^{3}+k U^{2}+C_{1}
$$

and the third order polynomial has three different roots. Taking into account Eq. (98), the solution of (67) for each root takes the form

$$
U=-\frac{5 k U_{0}+4 U_{0}^{2}+12 U_{0} \wp\left(\theta ; g_{2}, g_{3}\right)}{k+2 U_{0}-12 \wp\left(\theta ; g_{2}, g_{3}\right)}
$$

where

$$
g_{2}=\frac{k^{2}}{12}, \quad g_{3}=-\frac{k^{3}}{216}-\frac{C_{1}}{36} .
$$

If $C_{1} \neq 0$, the discriminant of $g_{2}$ and $g_{3}$ is different from zero, and therefore the solutions of Eq. (68) can not be simplified. Since we are interested in solitary wave and periodic solutions, we can choose $C_{1}=0$. Then, the invariants take the form of (29) with $n=1$ and $\Delta=0$. Thus, the third order polynomial has two different roots: 0 (twice) and $U_{0}=-3 k / 2$. While the root $U_{0}=0$ gives the trivial solution of Eq. (67), $U=0$, the nonzero root implies the following solution

or from (99)

$$
U=\frac{3 k}{4} \frac{2 k-12 \wp\left(\theta ; g_{2}, g_{3}\right)}{k+6 \wp\left(\theta ; g_{2}, g_{3}\right)}
$$

$$
U=6 \wp\left(\theta+\omega ; g_{2}, g_{3}\right)-\frac{k}{2}
$$

where $\omega$ is a half period. Now it is easy to check that (70) and (71) correspond to (30) and (31) with $n=1$, respectively. Therefore, the particular solutions of Eq. (2) can be found substituting $n=1$ in (36) and (37). 


\subsection{Modified BBM equation $(n=2)$}

Equation (11) with $n=2$ reduces to the modified BBM equation (3). Then, Eq. (58) becomes

$$
\frac{d^{2} U}{d \theta^{2}}-\left(U^{3}+3 \delta U^{2}+\left(\delta^{2}+k\right) U\right)=0
$$

with

$$
D_{2}=-k \delta-\delta^{3} .
$$

To obtain the solution of (172), we have to solve following first order equation that can be seen from the Section 2.3

$$
\frac{d U}{d \theta} \mp \sqrt{\frac{1}{2} U^{4}+2 \delta U^{3}+K U^{2}+C_{2}}=0
$$

where $K=3 \delta^{2}+k$. For this case we have the forth order polynomial

$$
f(U)=\frac{1}{2} U^{4}+2 \delta U^{3}+K U^{2}+C_{2}
$$

with four different roots. The solution of Eq. (74) for each root can be obtained, applying the same procedure mentioned above, as

$$
U(\theta)=\frac{5 K U_{0}+3 U_{0}^{3}+12 \delta U_{0}^{2}+12 U_{0} \wp\left(\theta ; g_{2}, g_{3}\right)}{12 \wp\left(\theta ; g_{2}, g_{3}\right)-k-3\left(\delta+U_{0}\right)^{2}}
$$

where

$$
g_{2}=\frac{K^{2}}{12}+\frac{C_{2}}{2}, \quad g_{3}=-\frac{K^{3}}{216}+\frac{C_{2} K}{12}-\frac{C_{2} \delta^{2}}{4},
$$

and $\Delta \neq 0$. Now, the solution of Eq. (3) can be obtained from

$$
u(x, t)=\sqrt{\frac{3 c}{a}}(U(\theta)+\delta) .
$$

If $C_{2}=0$ and $\delta \neq 0$, the forth order polynomial (75) has three different roots: 0 (with multiplicity two) and

$$
U_{0}^{ \pm}=-2 \delta \pm \sqrt{-2\left(k+\delta^{2}\right)} .
$$

In this case $\Delta=0$ and the invariants are

$$
g_{2}=\frac{K^{2}}{12}, \quad g_{3}=-\frac{K^{3}}{216} .
$$


When we substitute these roots and the degenerate forms of the Weierstrass function in Eq. (76), we have

$$
U^{ \pm}(\theta)=\frac{-2 K}{2 \delta \pm \sqrt{-2\left(k+\delta^{2}\right)} \cos [\sqrt{-K} \theta]}
$$

for $c>1$, and

$$
U^{ \pm}(\theta)=\frac{-2 K}{2 \delta \pm \sqrt{-2\left(k+\delta^{2}\right)} \cosh [\sqrt{K} \theta]}
$$

for $c<1$. Therefore, the solution of Eq. (3) can be obtained from (78) considering $k=(1-c) / c, \theta=x-c t$.

We can also deal with the case $C_{2} \neq 0, \delta=0$ and the corresponding forth order polynomial is

$$
f(U)=\frac{1}{2} U^{4}+k U^{2}+C_{2}
$$

has four different roots:

$$
U_{0}^{ \pm}= \pm \sqrt{-k-\sqrt{-2 C_{2}+k^{2}}}
$$

and

$$
U_{0}^{ \pm}= \pm \sqrt{-k+\sqrt{-2 C_{2}+k^{2}}}
$$

Substituting (81) in terms of $U_{0}$ in Eq. (98), the solution of Eq. (74) with $\delta=0$ for each root is

$$
U=-\frac{5 k U_{0}+3 U_{0}^{3}+12 U_{0 \wp}\left(\theta ; g_{2}, g_{3}\right)}{k+3 U_{0}^{2}-12 \wp\left(\theta ; g_{2}, g_{3}\right)}
$$

where

$$
g_{2}=\frac{k^{2}}{12}+\frac{C_{2}}{2}, \quad g_{3}=-\frac{k^{3}}{216}+\frac{C_{2} k}{12}
$$

and $\Delta \neq 0$.

Now, it is easy to see that the trivial choice $C_{2}=0$ gives rise to the invariants (41) with $n=2$ and $\Delta=0$. Then we will get the solitary wave and the periodic solutions. The roots $\left(U_{0}\right)$ also take the forms: 0 (multiplicity two) and $U_{0}^{ \pm}= \pm \sqrt{-2 k}$. Therefore, the Weierstrass function can be expressed in terms of hyperbolic and trigonometric functions. Then, the solitary wave and trigonometric solutions of Eq. (3) can be read from (36) and (37) with $n=2$ only for $U_{0}^{ \pm}$.

For this case, we have also another type of solution for certain values of $C_{2}=$ $k^{2} / 2$ for which we have the special form of (93): 
(1) If $c<1$, the equation

$$
\left(\frac{d U}{d \theta}\right)^{2}=\frac{1}{2}\left(U^{2}+k\right)^{2}
$$

has the solution

$$
U=\sqrt{k} \tan \left[\sqrt{\frac{k}{2}} \theta\right]
$$

(2) If $c>1$, the equation

$$
\left(\frac{d U}{d \theta}\right)^{2}=\frac{1}{2}\left(U^{2}-(-k)\right)^{2}
$$

has the solution

$$
U=\sqrt{-k} \tanh \left[\sqrt{\frac{-k}{2}} \theta\right] .
$$

Thus, for $c<1$ the solutions of (11) are periodic singular kink type

$$
u(x, t)=\sqrt{\frac{3(1-c)}{a}} \tan \left[\sqrt{\frac{1-c}{2 c}}(x-c t)\right]
$$

and for $c>1$ the solutions are of kink type

$$
u(x, t)=\sqrt{\frac{3(c-1)}{a}} \tanh \left[\sqrt{\frac{c-1}{2 c}}(x-c t)\right] .
$$

\section{Conclusions}

In this paper we have first factorized the generalized BBM equation in two ways. Then, we have investigated the travelling wave solutions of this equation by means of the factorization technique. We have obtained particular solutions of the generalized BBM as well as general solutions of the modified-BBM and BBM equations in terms of elliptic functions without making any ansatz [4,5,6]. We want to stress that this technique is more systematic than others previously used for the analysis of these equations. The factorization technique gives directly solutions of the BBM in terms of elliptic functions. Indeed, we have more general solutions and recovered all the solutions reported in [4,5,6]. At the same time we have shown the equivalence of certain expressions that, in fact, describe the same solutions for the BBM equation. 


\section{Acknowledgments}

This work has been partially supported by Spanish Ministerio de Educación y Ciencia (Projects MTM2005-09183, FIS2005-01375 and FIS2005-03989), Ministerio de Asuntos Exteriores (AECI grant 0000169684 of Ş.K.), and Junta de Castilla y León (Excellence Project VA013C05). Ş.K. acknowledges Department of Physics, Ankara University, Turkey, and the warm hospitality at De-

partment of Theoretical Physics, University of Valladolid, Spain, where this work has been carried out.

\section{Appendix: Elliptic functions}

Let us consider any quartic polynomial

$$
f(\varphi)=a_{0} \varphi^{4}+4 a_{1} \varphi^{3}+6 a_{2} \varphi^{2}+4 a_{3} \varphi+a_{4}
$$

and the differential equation

$$
\left(\frac{d \varphi}{d t}\right)^{2}=f(\varphi) .
$$

The simplest case in which Eq. (91) is integrable is when $f(\varphi)$ is given by

$$
f(\varphi)=\alpha_{0}^{2}\left(\alpha^{2} \pm \varphi^{2}\right)^{2} .
$$

Then the corresponding differential equation

$$
\left(\frac{d \varphi}{d t}\right)^{2}=\alpha_{0}^{2}\left(\alpha^{2} \pm \varphi^{2}\right)^{2}
$$

has the solutions

$$
\varphi^{-}=\alpha \tanh \left[\alpha \alpha_{0} \theta\right]
$$

and

$$
\varphi^{+}=\alpha \tan \left[\alpha \alpha_{0} \theta\right]
$$

In the general case, the invariants of (90) are defined as

$$
\begin{aligned}
& g_{2}=a_{0} a_{4}-4 a_{1} a_{3}+3 a_{2}^{2} \\
& g_{3}=a_{0} a_{2} a_{4}+2 a_{1} a_{2} a_{3}-a_{2}^{3}-a_{0} a_{3}^{2}-a_{1}^{2} a_{4}
\end{aligned}
$$

and the variable $z=\int_{\varphi_{0}}^{\varphi}[f(t)]^{-1 / 2} d t$, where $\varphi_{0}$ is any root of the equation $f(\varphi)=0$, is introduced. If the Weierstrass function $\wp\left(z ; g_{2}, g_{3}\right)$ is constructed 
with the help of the invariants $g_{2}$ and $g_{3}$, then $\varphi$ can be expressed as a rational function of it as

$$
\varphi=\varphi_{0}+\frac{1}{4} f^{\prime}\left(\varphi_{0}\right)\left(\wp\left(z ; g_{2}, g_{3}\right)-\frac{1}{24} f^{\prime \prime}\left(\varphi_{0}\right)\right)^{-1}
$$

where the prime $\left({ }^{\prime}\right)$ denotes the derivative with respect to $\varphi$. We have also the following useful relation for the Weierstrass function (once the invariants are fixed, and we can avoid them to alleviate the notation)

$$
\wp\left(z+\omega ; g_{2}, g_{3}\right)=e_{1}+\frac{\left(e_{1}-e_{2}\right)\left(e_{1}-e_{3}\right)}{\wp\left(z ; g_{2}, g_{3}\right)-e_{1}}
$$

where $\omega$ is half-period and $e_{1}, e_{2}, e_{3}$ are roots of the equation $4 t^{3}-g_{2} t-g_{3}=0$, such that

$$
\begin{aligned}
& e_{1}+e_{2}+e_{3}=0, \\
& e_{1} e_{2}+e_{1} e_{3}+e_{2} e_{3}=-\frac{1}{4} g_{2}, \\
& e_{1} e_{2} e_{3}=\frac{1}{4} g_{3} .
\end{aligned}
$$

When $g_{2}$ and $g_{3}$ are real and the discriminant

$$
\Delta=g_{2}^{3}-27 g_{3}^{2}
$$

is positive, negative or zero, we have different behaviors of $\wp(z)$.

Here, we shall discuss the case $\Delta=0$, that corresponds to degenerate cases of the Weierstrass functions which occur when one or both of periods become infinite, or, what is the same, two or all three roots $e_{1}, e_{2}, e_{3}$ coincide.

If $e_{1}=e_{2}=b>0, e_{3}=-2 b$, then $g_{2}>0, g_{3}<0$ and the real and imaginary periods of the Weierstrass function, $\omega$ and $\omega^{\prime}$, are $\omega=\infty, \omega^{\prime}=i \pi(12 b)^{-1 / 2}$, and this function can be written as

$$
\wp\left(z ; 12 b^{2},-8 b^{3}\right)=b+3 b \sinh ^{-2}\left[(3 b)^{1 / 2} z\right]
$$

which leads to solitary wave solutions.

If $e_{1}=2 b>0, e_{2}=e_{3}=-b$, then $g_{2}>0, g_{3}>0, \omega=\pi(12 b)^{-1 / 2}, \omega^{\prime}=i \infty$, and the Weierstrass function becomes

$$
\wp\left(z ; 12 b^{2}, 8 b^{3}\right)=-b+3 b \sin ^{-2}\left[(3 b)^{1 / 2} z\right],
$$

which leads to periodic solutions [10,11]. 


\section{References}

[1] Benjamin TB, Bona JL, and Mahony JJ. Model equations for long waves in nonlinear dispersive systems. Philos. Trans. R. Soc. London, Ser. A 1972;272:4778 .

[2] Clarkson PA. New similarity reductions and Painlevé analysis for the symmetric regularised long wave and modified Benjamin-Bona-Mahony equations. J. Phys. A: Math. Gen 1989;22:3821-48.

[3] Ince EL. Ordinary Differential Equations. New York: Dover; 1956.

[4] Wazwaz A-M. New travelling wave solutions of different physical structures to generalized BBM equation. Phys Lett A 2006;355:358-62.

[5] Wazwaz A-M, and Helal MA. Nonlinear variants of the BBM equation with compact and noncompact physical structures. Chaos, Solitons and Fractals $2005 ; 26: 767-76$.

[6] Nickel J. Elliptic solutions to a generalized BBM equation.Phys Lett A 2006;364:221-28.

[7] Tang Y, Xu W, Gao L, and Shen J. An algebraic method with computerized symbolic computation for the one-dimensional generalized BBM equation of any order. Chaos, Solitons and Fractals 2007;32:1846-52.

[8] Estévez PG, Kuru Ş, Negro J, and Nieto LM. Travelling wave solutions of twodimensional Korteweg-de Vries-Burgers and Kadomtsev-Petviashvili equations. J Phys A: Math Gen 2006;39:11441-52.

[9] Cornejo-Pérez O, Negro J, Nieto LM, and Rosu HC. Travelling-wave solutions for Korteweg-de Vries-Burgers equations through factorization. Found Phys 2006;36:1587-99.

[10] Erdelyi A et al (Eds.). The Bateman Manuscript Project. Higher Transcendental Functions Volume II. Malabar, FL: Krieger Publishing Co.; 1981.

[11] Whittaker ET and Watson G. A Course of Modern Analysis. Cambridge: Cambridge University Press; 1988. 